\title{
Profiles of Diabetic Ketoacidosis in Multiethnic Diabetic Population of Malaysia
}

\author{
Atif Usman ${ }^{1,2 *}$, Syed Azhar Syed Sulaiman ${ }^{2}$, Amer Hayat Khan ${ }^{2,3}$ and Azreen \\ Syazril Adnan ${ }^{3}$ \\ ${ }^{1}$ Department of Pharmacy Practice, Faculty of Pharmaceutical Sciences, Government College University Faisalabad, 38100 \\ Faisalabad, Pakistan, ${ }^{2}$ Discipline of Clinical Pharmacy, School of Pharmaceutical Sciences, University of Science Malaysia, \\ 11800 Penang Island, Malaysia, ${ }^{3}$ Chronic Kidney Disease (CKD) Resource Center, School of Medical Sciences, University of \\ Science Malaysia, 16150 Kota Bharu, Kelantan, Malaysia
}

*For correspondence: Email: miyan.atif@gmail.com

\begin{abstract}
Purpose: To outline first-time patient profiles of diabetic ketoacidosis (DKA) in the absence of reported incidence and mortality rates of DKA in Malaysian diabetic population.

Methods: A retrospective cross-sectional study was designed and all medical records of patients with a discharge note of DKA were reviewed. Admissions from January 2009 to December 2011 were included. Data were analyzed in terms of socio-demographic variables in order to provide incidence and mortality rates. Medical history, as well as physical and biochemical characteristics were analyzed to report epidemiology of DKA patients.

Results: Out of a total of 207 admissions for DKA, 132 were selected for the present study. Female (62.9\%), Malay ethnic (47.0\%) and the elderly (45.1 years and above) contributed most to DKA episodes. Type 2 diabetes mellitus $(51.1 \%)$ patients were prone to develop DKA. Most patients experienced mild to moderate episode of DKA by the time they sought medical attention. Although, there was no significant relationship between chronic co-morbidity and occurrence of DKA, hypertension (54.5\%), dyslipidemia (43.0\%) and cardiac disorders (35.6\%) were, however, the most frequently observed co-morbidities. Non-adherence (43.2 \%), sepsis (31.9\%) and respiratory tract infection (12.2 $\%)$ were the most encountered precipitating factors for DKA episode. Mortality rate was as high as 17.6 $\%$.

Conclusion: With a higher incidence and mortality rate of DKA in Malaysia, the patterns observed in this study seem to be different from those of developed nations. Further extended studies need to be undertaken to elaborate regional and national patterns of DKA.
\end{abstract}

Keywords: Incidence, Mortality, Diabetic ketoacidosis, Diabetes, Hypertension, Cardiac disorders, Dyslipidemia, Comorbidity

Tropical Journal of Pharmaceutical Research is indexed by Science Citation Index (SciSearch), Scopus, International Pharmaceutical Abstract, Chemical Abstracts, Embase, Index Copernicus, EBSCO, African Index Medicus, JournalSeek, Journal Citation Reports/Science Edition, Directory of Open Access Journals (DOAJ), African Journal Online, Bioline International, Open-J-Gate and Pharmacy Abstracts

\section{INTRODUCTION}

Diabetic ketoacidosis (DKA) is an acute complication of diabetes mellitus (DM) that was incurable before discovery of insulin [1]. After successful incorporation of insulin in DM management, and with exclusive studies carried out to improve understanding of DKA, incidence and mortality rates of DKA kept decreasing [2]. The incidence was once reported to be as low as 4.6 - 8.0 DKA episodes per 1000 diabetic admissions with a mortality rate of $5 \%$ [3]. However, this trend is now followed to be increasing in recent studies. Incidence rate of 
DKA increased from 8.5 to 15.6 per 100,000 per year whereas mortality rate increased from 1.5 to $4.0 \%$ in Danish population over a period of 20 years [4]. Moreover, DKA remains a constant burden on mortality and morbidity among DM patients.

Contrary to the extensive worldwide concern on DKA, to date, there has been only one published study from Malaysia that was carried out with specific objectives based on the biochemical differences among the types of DM which did not provide any incidence or mortality rate, however [5]. It was further noted that there has not been any developed protocol provided to the hospitals on national level in Malaysia for management of DKA. Hence, we intended to seek the incidence of DKA in Malaysian population, and further aimed to evaluate the clinical practices and treatment outcomes of the physicians in absence of any integrated care pathway.

\section{EXPERIMENTAL}

Patient profiles from a public hospital from Northern Malaysia peninsula were retrospectively sought for period of January 2009 to December 2011. All hospitalizations against diabetic admissions and endocrine referral were reviewed. Discharge code for DKA, i.e., E1X.1 was followed in accordance with International Classification of Diseases v.10 (ICD-10) where X ranged from $0-4$ [4]. Hospital admission was attributable as DKA for patient with arterial $\mathrm{pH}$ less than 7.30 , hyperglycemia and positive ketones in either serum or urine. Notably, euglycemic episodes of ketoacidosis were also included. An episode was also considered as DKA where $\mathrm{HCO}_{3}^{-}$was recorded equal or less than $18 \mathrm{mmol} \cdot \mathrm{L}^{-1}$ with an arterial $\mathrm{pH}$ value of 7.30-7.35 [6,7]. Provided that venous blood sample was drawn for initial diagnosis, 0.03 and 0.52 mmol. $\mathrm{L}^{-1}$ was added for $\mathrm{pH}$ and $\mathrm{HCO}_{3}{ }^{-}$, respectively [8]. Social demographics, and physical and biochemical profiles were recorded for incidence and endpoint focused results.

Patients were classified as T1DM, T2DM and NDDM on the basis of their medical record. Diagnosis of DM at the time of admission for DKA management rendered patient as NDDM. Patients that were managed with oral antidiabetic (OAD) medication over the span of their diabetic morbidity were tagged as T2DM. Similarly, NDDM patients managed with OAD or life style modification at the time of discharge or during follow up at diabetic clinic were considered as T2DM.

\section{Data analysis}

Statistical Package for Social Sciences (SPSS) ( $\mathrm{V}$.16 was used to analyze the acquired data. With a confidence interval of $95 \%$, results were considered significant $(p \leq 0.05)$. Moreover, continuous data are expressed as mean \pm standard deviation (SD), and categorical data as number/percentage ( $\mathrm{N} / \%)$.

\section{Ethical approval}

The study was registered with, and approved by National Medical Research Register (NMRR), Malayisa.

\section{RESULTS}

Total 2536 patients were referred to in-patient facility over a period of 3 years for management of DM related disorder. Out of these, 207 admissions were found with a discharge note of DKA. On the basis of inclusion criteria, 142 patient records were selected for this study out of which, 25 were fatalities. Ten files of deceased patients were not retrievable and hence, present study is concluded on 132 patients (Figure 1).

\section{Incidence rate and socio-demographics}

Of total 132 hospital admissions studied for DKA (Table 1), nearly two-thirds were recorded in female diabetes patients $(62.9 \%)$. Mean age of patients was $49.7 \mathrm{yrs} \pm$ (16.3) while least admissions $(16.7 \%)$ were observed in patients that were in their middle age (25.1 to $45 \mathrm{yrs}$ ). Most admissions were constituted by Malay patients $(47.0 \%)$ followed by Chinese and Indians (31.8 and $19.7 \%$, respectively).

\section{Epidemiological findings}

More than half patients $(51.5 \%)$ were established case of type 2 DM in our study as depicted in Table 2. Notably, $12.1 \%$ patients were diagnosed with DM upon their hospital admissions. Among chronic co-morbidities, 54.5 $\%$ patients were found to accompany hypertension, $43.0 \%$ patients with dyslipidemia and $35.6 \%$ patients with cardiovascular disorder. We further observed $9.1 \%$ episodes of DKA in patients with thyrotoxicosis. Around half (52.3\%) of the patients had 2 or more associated comorbidities. Meanwhile, frequently observed acute co-morbidities were sepsis (31.9\%), respiratory tract infection (12.2\%) and gastric disorders $(8.4 \%)$. 


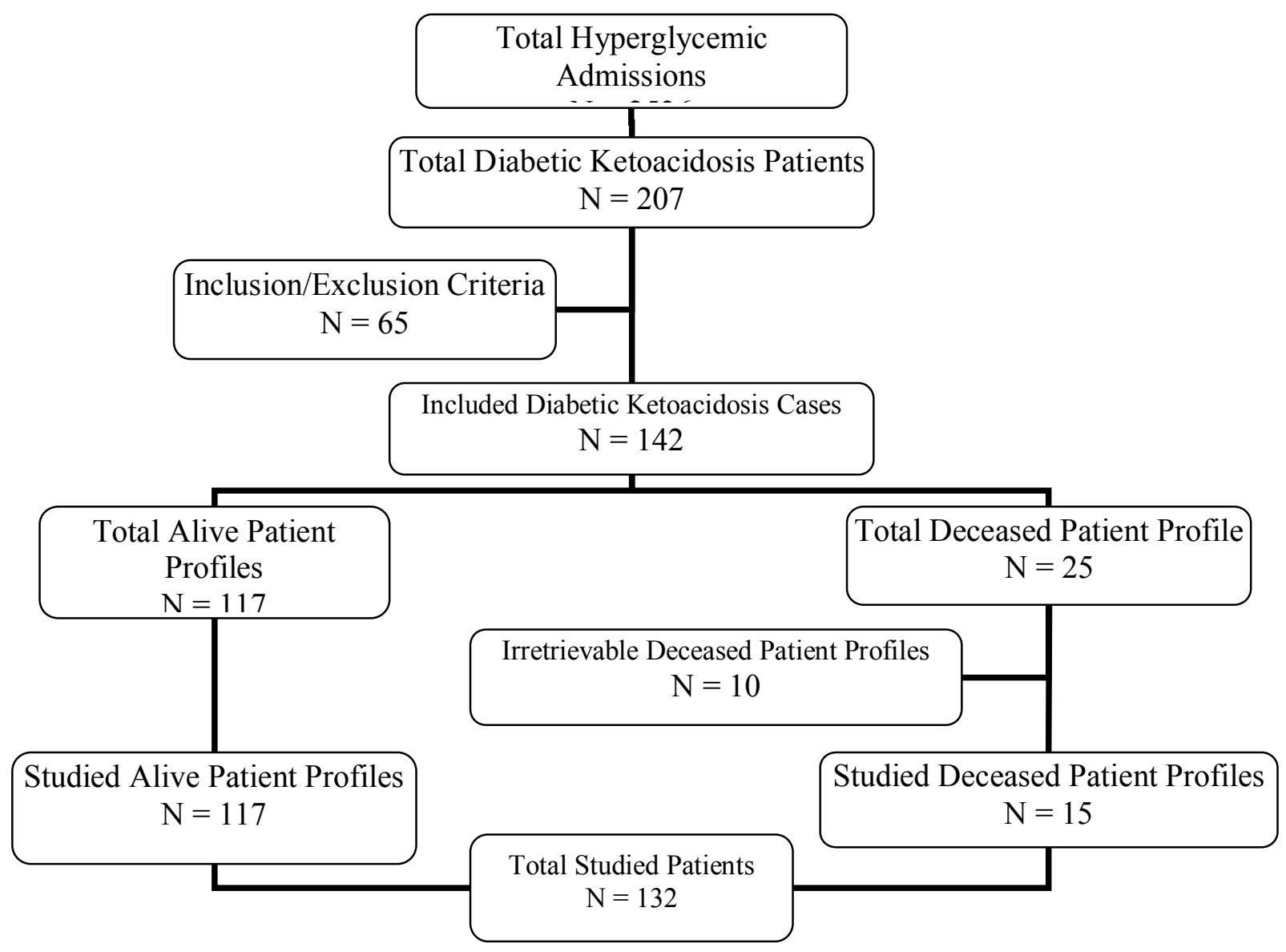

Figure 1: Flow chart of the selected one hundred and thirty two patient profiles

Table 1: Socio-demographic characteristics of DKA patients $(\mathrm{N}=132)$

\begin{tabular}{lc}
\hline Characteristic & Number (\%) \\
Gender & \\
- Male & $49(37.1)$ \\
- Female & $83(62.9)$ \\
Age mean \pm (SD) years & $49.7 \pm(16.3)$ \\
Age group (years) & \\
- $\quad 18-25$ & $17(12.9)$ \\
- $25.1-30$ & $5(3.8)$ \\
- $30.1-35$ & $8(6.1)$ \\
- $35.1-40$ & $2(1.5)$ \\
- $40.1-45$ & $7(5.3)$ \\
- $45.1-50$ & $19(14.4)$ \\
- $50.1-55$ & $19(14.4)$ \\
- $55.1-60$ & $21(15.9)$ \\
- 60.1 and above & $34(25.8)$ \\
Ethnicity & \\
- Malay & \\
- Chinese & $62(47.0)$ \\
- Indian & $42(31.8)$ \\
- Other & $26(19.7)$ \\
Deceased & $2(1.5)$ \\
\hline
\end{tabular}

${ }^{\dagger}$ Based on 132 patient profiles; however, total number of deceased patients was 25 (17.6 \%) based on 142 patient profiles

\section{Physical and biochemical presentation}

Nausea and vomiting were reported by most $(62.4 \%)$ DKA patients followed by the constitutional symptoms (51.6\%), which include dizziness, loss of appetite, lethargy and fever. Sign of dehydration was recorded in $83.3 \%$ patients whereas cardiac disorders in nearly one third $(31.9 \%)$ of the DKA patients. Least observed sign i.e. ketotic breath was documented for almost $20 \%$ of patients. On average, patients were afebrile $\left(37.1 \pm 0.8{ }^{\circ} \mathrm{C}\right)$ with isolated systolic hypertension $(130 \pm 27$ $\mathrm{mmHg}$ ) by the time they sought medical advice.

Blood glucose $\left(30.4 \pm 13.3 \mathrm{mmol} \cdot \mathrm{L}^{-1}\right)$ and ketones' $\left(4.6 \pm 1.7 \mathrm{mmol} \cdot \mathrm{dL}^{-1}\right)$ levels were found to be elevated with lowered arterial $\mathrm{pH}$ $(7.15 \pm 0.16)$ and bicarbonate $\left(9.7 \pm 5.5 \mathrm{mmol} . \mathrm{L}^{-}\right.$ $\left.{ }^{1}\right)$. By further dividing the patients on the basis of severity of acidosis, most $(42.4 \%)$ of them presented with moderate episode of DKA. Potassium level $\left(4.3 \pm 1.2 \mathrm{mmol} . \mathrm{L}^{-1}\right)$, and hematology profile except for white blood count $\left(17.8 \pm 7.5 \times 103 . \mu \mathrm{L}^{-1}\right)$, was also found to be within the normal limits (Table 4). 
Table 2: Epidemiological findings against admissions associated with DKA $(\mathrm{N}=132)$

\begin{tabular}{lc}
\hline Variable & N (\%) \\
\hline Type of DM & \\
- Type 1 DM & $48(36.4)$ \\
- Type 2 DM & $68(51.5)$ \\
- Newly diagnosed & $16(12.1)$ \\
Chronic co-morbidities ${ }^{\dagger}$ & \\
- Hypertension & $72(54.5)$ \\
- Dyslipidemia & $56(43.0)$ \\
- Cardiovascular Disorder & $47(35.6)$ \\
- Renal Disorder & $5(4.6)$ \\
- Thyrotoxicosis & $12(9.1)$ \\
- Other & $19(14.4)$ \\
- None & $37(28.0)$ \\
Multiple chronic co-morbidity & \\
- Single chronic co-morbidity & $26(19.7)$ \\
- Multiple chronic co-morbidities & $69(52.3)$ \\
- None & $37(28.0)$ \\
Acute co-morbidity & \\
- $\quad$ Sepsis & \\
- Respiratory tract infection & $42(31.9)$ \\
- Gastric disorder & $16(12.2)$ \\
- $\quad$ Acute renal failure & $11(8.4)$ \\
- Diabetic foot ulcer & $9(6.8)$ \\
- Urinary tract infection & $5(3.8)$ \\
- None & $3(2.3)$ \\
Positive pathological smear & $57(43.2)$ \\
\hline
\end{tabular}

${ }^{\dagger}$ Should a single patient present none or more than one acute/chronic co-morbidity; numerals hereby given as number (\%) are not to be tallied with total number of patients $(N=132)$

\section{DISCUSSION}

Prevalence of diabetes mellitus (DM) is increasing worldwide and the situation is much critical in developing nations which contribute the most in global burden of DM [9]. A high prevalence rate is observable in most of the developing nations. Similar findings were also provided by ministry of health $(\mathrm{MOH})$ Malaysia with a prevalence rate of $11.6 \%$ for DM [10].

Such burden includes DM associated chronic and acute co-morbidities as well. Being an acute co-morbidity, the recorded incidence rate for DKA varies from 4.6 to 8 out of 1000 diabetic admissions in developed nations [3]. However, it is reported to be higher in Asia, Africa and Polynesia [11-13]. Huri et al reported 256 DKA cases over 5 years in Malaysia that was slightly higher when compared with neighboring countries [5]. As per patient profiles analyzed in our study, we estimated incidence rate of DKA to be $5.47 \%$ (54.7 per 1000 diabetic admissions) for multiethnic diabetic population of Malaysia.
Table 3: Physical presentation of DKA patients at the time of admission $(\mathrm{N}=132)$

\begin{tabular}{lc}
\hline Variable & N (\%) \\
\hline Symptom & $81(61.4)$ \\
- Nausea/Vomiting & $68(51.6)$ \\
- Constitutional Symptoms & $59(44.7)$ \\
- Polyuria/Polydipsia & $50(37.8)$ \\
- Shortness of Breath & $41(31.0)$ \\
- Abdominal Pain & \\
Sign & $110(83.3)$ \\
- Dehydration & $64(48.4)$ \\
- Kussmaul breathing & $55(41.7)$ \\
- Constitutional signs & $42(31.9)$ \\
- Cardiac disorder & \\
- Ketotic breath & $26(19.7)$ \\
Temperature ( $\left.{ }^{\circ} \mathbf{C}\right)$ & $37.1 \pm(0.8)$ \\
Blood pressure systolic $(\mathbf{m m H g})$ & $130 \pm(27)$ \\
Blood pressure diastolic $(\mathbf{m m H g})$ & $74 \pm(19)$ \\
Pulse rate (beats/min) & $111 \pm(23)$ \\
\hline
\end{tabular}

Table 4: Biochemical and hematological profiles of DKA patients at the time of admission $(\mathrm{N}=132)$

\begin{tabular}{lc}
\hline Variable & Value \\
\hline Blood Glucose Level mmol/L & $30.4 \pm(13.3)$ \\
Ketones $\mathrm{mmol} / \mathrm{dL}$ & $4.6 \pm(1.7)$ \\
Arterial $p \mathrm{H}$ & $7.15 \pm(0.16)$ \\
Severity of DKA Episode $\mathrm{n}(\%)$ & \\
- Mild & $48(36.4)$ \\
- Moderate & $56(42.4)$ \\
- Severe & $28(21.2)$ \\
Arterial Bicarbonate $\mathrm{mmol} / \mathrm{L}$ & $9.7 \pm(5.5)$ \\
Serum Sodium $\mathrm{mmol} / \mathrm{L}$ & $130.4 \pm(7.6)$ \\
Serum Potassium $\mathrm{mmol} / \mathrm{L}$ & $4.3 \pm(1.2)$ \\
WBC Count $\times 10^{3} / \mu \mathrm{L}$ & $17.8 \pm(7.5)$ \\
RBC Count $\times 10^{6} / \mu \mathrm{L}$ & $4.6 \pm(0.9)$ \\
Hemoglobin $\mathrm{g} / \mathrm{dL}$ & $12.9 \pm(2.6)$ \\
Hematocrit $\%$ & $40.9 \pm(24.2)$ \\
Platelets $\times 10^{3} / \mu \mathrm{L}$ & $288 \pm(135)$ \\
\hline
\end{tabular}

We found this rate to be parallel with the estimated rate of DKA for Malaysia and reported rates for Thailand and China $[5,12,13]$.

Diabetic ketoacidosis is dominantly found in young and old patients and least admissions are observed in middle-aged patients. Such observation is due to occurrence of T1DM in children and youngsters. It is further observed as "Maturity Onset of Diabetes in Young" (MODY) in adolescent patients. Moreover, old aged patients are likely to develop T2DM. In both the age groups, it is difficult to deal with a sudden lifestyle modification [14]. Similar trend was also found in our research where we recorded only 22 cases in patients with age ranging between 25.1 and 45 years. We also found that nearly half $(47.0 \%)$ 
admission profiles were accredited to Malay patients and to an extent, Malay females were most prone to develop DKA $(\mathrm{N}=37)$. Nevertheless, it is not possible to compare these findings with any published data.

Diabetic ketoacidosis was once considered a complication of insulin dependent diabetic patients [1]. With passage of time, it was also found to be associated with T2DM and NDDM patients when numbers of these patients were reported to present with DKA [15]. In recent past, more studies reporting similar findings in such context give a notion that although the incidence of DKA in T2DM may be less, but since prevalence of T2DM exceeds T1DM, DKA is thus more or less observed equally in both arms [12]. Interestingly, various studies conducted in developed countries report occurrence of DKA in T1DM patients $[3,4,15]$. Contrarily, the studies conducted in Asia, South East Asia and Polynesia, reported T2DM to be more common among the patients of DKA $[12,13]$. Parallel with the regional findings, Huri et al reported frequent occurrence of DKA in T2DM patients [5]. We also found DKA to be associated with T2DM for which, nearly half patients were established cases of T2DM in our study. Moreover, among the NDDM patients, only 3 were found to be T1DM patients. However, this gesture is based on discharge medication and medical follow up notes, and neither includes plasma C-peptide activity nor islet cell autoimmunity assay [16].

Lifelong co-morbidities of metabolic continuum are mostly associated with DM. To an extent, if DM indirectly influences development of macrovascular complications, microvasular complications are directly related with poor glycemic control, however [14]. In our study, hypertension was the accompanied co-morbidity in more than half DKA patients. It was followed by dyslipidemia and cardiovascular disorders. Contrarily, renal disorder being a leading cause of DKA induced mortality, we only located 5 and 9 patients that accompanied chronic and acute renal failure at the time of admission, respectively $[4,17]$. On the other hand, influence of thyroid hormones on lipid metabolism and insulin counter regulatory hormones render thyroroxicosis as a mild risk factor for development of DKA [18]. We, however, recorded 19 established cases of thyrotoxicosis patients in our study. Among the precipitating factors for an episode of DKA, infection and nonadherence to DM management are, more or less, equally reported $[5-7,12,13]$. In our research, most patients did not have any impulsive cause for development of DKA. Sepsis was recorded as the foremost cause which was followed by respiratory tract infection. Although a high number of patients $(\mathrm{N}=75)$ were perceived as carrying any infection in our study, only 40 (30.4 $\%$ patients were found with a positive pathological smear throughout their hospitalization.

Hyperglycemia, ketones and acidosis are contributing factors for development of DKA. These factors have their own distinguished sign and symptoms; hyperglycemia causes polyurea, polydipsia and dehydration; shortness of breath, tachypnoea and Kussmaul breathing are distinct feature of ketosis; severe acidosis may induce abdominal pain, decreased sensorium and intruding focus. Nevertheless, most of the symptoms and signs are overlapped by all three factors [6]. Foremost abnormality induced by hyperglycemia is shift of water, and in turn, of important physiologic ions from intracellular to extracellular space to maintain osmotic pressure. Among these, sodium and potassium play important role in physical presentation of a DKA patient. Initial hypernatremia causes lethargy and diuresis whereas hyperkalemia, by irritation of gastric mucosa, induces nausea and vomiting. On the other hand, production of ketones and deterioration of $\mathrm{pH}$ also propagate abdominal and respiratory distress. Dehydration is the second effect due to hyperglycemia driven diuresis. It causes hypovolemia induced peripheral vasodilation which triggers hypothermia, a distinct character of DKA that is a reaction to peripheral vasodilation and hypovolemia [6,7]. These signs and symptoms exacerbate with progress of DKA episode and encompass deterioration of cardiac and cerebral function as well $[6,7,18]$. In our research, chief complaint registered by patients was nausea and vomiting, followed by constitutional symptoms and polyurea \& polydipsia. Similarly, dehydration was the most observed sign reported by attending physicians on the basis of decreased skin turgor and dried mucous membrane, followed by constitutional signs $[6,18]$. Nearly one third of patients were found with acute cardiac abnormality at the time of admission in our study. Apart from key factors of DKA, studies indicate that systolic blood pressure decreases up to $90 \mathrm{mmHg}$ while pulse rate increase up to 120 beats $\cdot \mathrm{min}^{-1}$ in DKA $[6,18]$. Though patients in our research were observed with an elevated pulse rate, they however were found with an isolated systolic hypertension and diastolic eutension. Furthermore, with around $80 \%$ of patients to be dehydrated upon the initial physical examination, patients were observed as euthermic on average at the time of admission. Such findings, however, could only be reasoned 
on the basis of their accreditation to severity of acidosis [19].

Both BGL and ketones are the founding character of DKA and have an important impact on identification and discharge of DKA patient. Effects of hyperglycemia also reciprocate in hematology profile of a DKA patient. Such effects are mainly due to hyperglycemia induced dehydration and cause an increase in leukocyte count, red blood cell count and hematocrit [20]. This value, however, could not be interpreted to infection as designated value for presence of infection in DKA is considered to be above $25.0 \mathrm{x}$ $10^{3} \cdot \mu \mathrm{L}^{-1}[6,7,18]$. Moreover, BGL may vary from 3 to $50 \mathrm{mmol} \cdot \mathrm{L}^{-1}$ whereas presence of ketones in either urine or serum is enough to authenticate DKA. This leaves arterial $\mathrm{pH}$ and bicarbonate levels to be chief indicator for severity of DKA $[6,7,15,18]$. Although values for blood glucose and ketones' were higher at the time of admission in our study, the recorded mean arterial $\mathrm{pH}$ and bicarbonate level of patients reciprocate moderate episode of DKA $[6,7]$. Upon further division of patients on the basis of severity of the episode, we found that most admissions ranger from mild to moderate DKA. Likewise, mean WBC was found to be elevated contrary to red blood count and hematocrit, which on average were found to be well within range. We further find most of our observations to be parallel with similar studies $[11,12]$.

Mortality rate for DKA was $90 \%$ before the discovery of insulin and after the successful intervention in management of T1DM, it was significantly reduced to $14.5 \%$ by the mid of 20th century [2]. Among developed nations, the mortality rate in Denmark was reported to be $4 \%$ in 2007 [4]. However, it remains higher for developing countries. Study conducted in Asia Pacific region found a fatality rate of $10 \%$ and $11.8 \%$ [16]. In context of mortality, $11.3 \%$ patients, out of 132 , did not survive the episode of DKA in present study (17.6\%, if based on 142 patient profiles). Meanwhile, due to unavailability of any reported casualty rate for DKA in Malaysia, it was also found to be higher in this study when compared with the worldwide and regional mortality rates.

\section{Limitations of the study}

The study was retrospective. Importantly, the total number of deceased patients' profiles ( $\mathrm{N}=$ 25 ) was not available for review and hence, the study could not significantly dwell on bio- or therapeutic-markers that may cause fatal outcome. The limited number of patients used meant that no correlation could be determined between deviation from standard treatment guideline and altered serum electrolytes in discharged patients.

\section{CONCLUSION}

Our findings suggest that profiles of diabetic ketoacidosis patients are different for western and eastern parts of the world. Additionally, DKA remains a frequently observed hyperglycemic emergency with a high incidence and fatality rate in multiethnic Malaysian population. Sociodemographic results from current study propose that females, Malays, and adolescent and older patients of diabetes mellitus are more prone to develop DKA. Similarly, epidemiological results depict DKA to be associated more with type 2 than type $1 \mathrm{DM}$, and hypertension and infection are more commonly found chronic and acute comorbidities, respectively. Nevertheless, most of our findings could only be authenticated when a large scale research is undertaken.

\section{REFERENCES}

1. Dreschfeld J. The Bradshawe Lecture on Diabetic Coma. Brit Med J 1886; 2: 358-363.

2. Skillman TG, Wilson R, Knowles HC Jr. Mortality of patients with diabetic acidosis in a large city hospital. Diabetes 1958; 7(2): 109-113.

3. Faich GA, Fishbein HA, Ellis SE. The epidemiology of diabetic acidosis: a population-based study. Am J Epidemiol 1983; 117(5): 551-558.

4. Henriksen OM, Roder ME, Prahl JB, Svendsen OL. Diabetic ketoacidosis in Denmark Incidence and mortality estimated from public health registries. Diabetes Res Clin Pr 2007; 76(1): 51-56.

5. Huri HZ, Foong GTK, Pendek R, Widodo RT. Different characteristics of diabetic ketoacidosis between type 1 and type 2 diabetes patients in Malaysia. Asian Biomed 2009; 3(2): 201-205.

6. Kitabchi AE, Umpierrez GE, Murphy MB, Kreisberg RA. Hyperglycemic crises in adult patients with diabetes: a consensus statement from the American Diabetes Association. Diabetes Care 2006; 29(12): 2739-2748.

7. Savage MW, Dhatariya KK, Kilvert A, Rayman G, Rees $J A$, Courtney $\mathrm{CH}$, Hilton L, Dyer PH, Hamersley MS. Joint British Diabetes Societies guideline for the management of diabetic ketoacidosis. Diabetic Med 2011; 28(5): 508-515.

8. Middleton $P$, Kelly AM, Brown J, Robertson M. Agreement between arterial and central venous values for $\mathrm{pH}$, bicarbonate, base excess, and lactate. Emerg Med J 2006; 23(8): 622-624.

9. Shaw JE, Sicree RA, Zimmet PZ. Global estimates of the prevalence of diabetes for 2010 and 2030. Diabetes Res Clin Pr 2010; 87(1): 4-14. 
10. Letchuman GR, Wan Nazaimoon WM, Wan Mohamad WB, Chandran LR, Tee GH, Jamaiyah $H$, Isa MR, Zanariah H, Fatanah I, Ahmad Faudzi Y. Prevalence of diabetes in the Malaysian National Health Morbidity Survey III 2006. Med J Malaysia 2010; 65(3): 180-186.

11. Elmehdawi RR, Elmagerhei HM. Profile of diabetic ketoacidosis at a teaching hospital in Benghazi, Libyan Arab Jamahiriya. East Mediterr Health J 2010; 16(3): 292-299.

12. Tan $H$, Zhou $Y, Y u Y$. Characteristics of diabetic ketoacidosis in Chinese adults and adolescents - $A$ teaching hospital-based analysis. Diabetes Res Clin Pr 2012; 97(2): 306-312.

13. Thewjitcharoen $Y$, Sunthornyothin S. Clinical characteristics of diabetic ketoacidosis in newly diagnosed adult patients. Diabetes Res Clin Pr 2010; 90(2): 43-45.

14. Holt TA, Kumar S, Watkins PJ. ABC of diabetes. 6th edn. $A B C$ series. 2010, Chichester, West Sussex, UK; Hoboken, NJ: Wiley-Blackwell/BMJ. viii, 104.

15. Westphal SA. The occurrence of diabetic ketoacidosis in non-insulin-dependent diabetes and newly diagnosed diabetic adults. Am J Med 1996; 101(1): 19-24.
16. Chu CH, Lee JK, Lam HC, Lu CC. The Occurrence of Diabetic Ketoacidosis in Type 2 Diabetic Adults, 1997 [cited 2011 Dec 15]. Available from: http://www.tsim.org.tw/journal/jour106/P10_230.PDF.

17. Barski L, Harman-Boehm I, Nevzorov R, Rabaev E, Zektser M, Jotkowitz AB, Zeller L, Shleyfer E, Almog $Y$. Gender-Related Differences in Clinical Characteristics and Outcomes in Patients with Diabetic Ketoacidosis. Gender Med 2011; 8(6): 372377.

18. Stewart C. Diabetic Emergencies: Diagnosis And Management Of Hyperglycemic Disorders. Emerg Med Pract 2004; 6(2):1-24.

19. Tokuda Y, Omata F, Tsugawa Y, Maesato K, Momotura $K$, Fujinuma A, Stein GH, Cook EF. Vital sign triage to rule out diabetic ketoacidosis and non-ketotic hyperosmolar syndrome in hyperglycemic patients. Diabetes Res Clin Pr 2010; 87(3): 366-371.

20. Barone B, Rodacki M, Cenci MC, Zajdenverg L, Milech A, Oliveira JE. Diabetic ketoacidosis in adults--update of an old complication. Arq Bras Endocrinol \& Metab 2007; 51(9): 1434-1447. 